\title{
Context-Aware Adaptation of User Interfaces
}

\author{
Vivian Genaro Motti ${ }^{1}$, Jean Vanderdonckt ${ }^{1}$ \\ ${ }^{1}$ Université catholique de Louvain, Louvain School of Management \\ Louvain Interaction Laboratory, Place des Doyens 1 \\ B-1348 Louvain-la-Neuve, Belgium \\ \{vivian.genaromotti,jean.vanderdonckt\}@uclouvain.be
}

\begin{abstract}
Efficient adaptation aims at ensuring that a user interface is adapted to a user's task according to the context of use, since the end user is carrying out a task with one or several computing platforms in a physical environment. This tutorial presents key concepts of adaptation: principles that guide it, relevant context information and how to consider it, dimensions and abstraction levels subject to adaptation, as well as, languages, methods and techniques used in this domain. This tutorial aims at teaching major aspects to be considered for adaptation of user interfaces in general and concerning the context of use in particular, including the end user (or several of them, as in multi-user interfaces), the platform (or several of them, as in multi-device environments), and the physical environment (or several of them, as in multi-location systems).
\end{abstract}

Keywords: Multi-dimensional adaptation; Context-aware adaptation; Adaptive applications; Adaptable applications.

\section{Tutorial Information}

Adaptation changes system aspects according to its context and defined rules. Many dimensions can be subject to it (as navigation and presentation), and in different granularity levels, ranging from atomic units to the whole application. Efficient adaptation provides users a better interaction by considering the context. It may be performed by rules and supported by methods, techniques, languages and machine learning approaches. Methods combine adaptation techniques, e.g. to adapt the content, a text can be summarized, simplified and described. Languages that respect separation of concerns (user interface description languages) are recommended. The application domains for adaptation vary: entertainment, education, e-commerce. Contributions in this field have been reported since the early 90's, yet, the evolution of the technologies requires constant efforts to provide adaptation efficiently. Also, current users interact with new devices (e.g. idTV), via different means (e.g. touchscreens), using new technologies (e.g. RIAs). The varied contexts, dimensions and levels for adaptation are a challenge. Users may feel confused or overloaded with excesses. Today, a large body of knowledge about adaptation exists but it is very scattered and widespread in the literature, requiring time and efforts to find relevant information when one wishes to support some form of user interface adaptation 
(adaptability and/or adaptivity). This tutorial addresses this need providing an overview of user interface adaptation in general and of context-aware adaptation in particular. It covers the state-of-the-art of multi-dimensional context-aware adaptation, in scientific and commercial domains, and it is organized in:

Fundamental Concepts. The diversity of contexts of use is a challenge for the development of systems that suits to user' preferences, profiles, and requirements. Designing different versions of a system for each scenario is a possible strategy to perform adaptation, though it demands effort and may lead to inconsistent results. Models of different abstraction levels can also be created to accommodate varied scenarios [1]. Adaptation aims at a universal access, independent of user, platform, and environment. The context of use is the source of information for adaptation, it can be automatically or manually gathered. Many dimensions of a system can be subject to adaptation, and in different granularity levels. The context defines the priority of each dimension. Principles, as plasticity, rules and techniques support this process.

Theoretic Models. Taxonomies and ontologies have been used to classify, define and model context, and adaptation techniques. The context-aware design space (CADS) organizes dimensions and levels for adaptation. Orthogonal axis defines granularity levels for each dimension. Adaptive/adaptable applications can be compared with CADSs, which are descriptive, extensible and flexible. A reference framework organizes context information for adaptation, defining where, when, how adaptation can be performed. To adapt system resources many techniques can be applied, a template details them. Adaptation models describe views of a system, and the recommended languages in this sense, consider separation of concerns. Animation is one possible strategy to present the adaptation result smoothly for users [2].

Use Cases. In commercial domain examples of application include: (i) the Google search engine that adapts the order of the results according user preferences; Google AdSense that provides context-aware advertisements; (ii) Amazon recommends products according to the shops of other costumers; (iii) NYTimes suggests articles according to their content; and (iv) Nokia Situations that allows users to define context settings for device acts. In scientific domain, examples include: (i) The Sedan-Bouillon website [3] that adapts its content according to the platforms and user preferences, (ii) QTk Draw [4] that allows users to distribute the application in different devices, and (iii) ELM-ART [5] that adapts the navigation for the user.

\section{References}

1. Motti, V. G. A Computational Framework for Multi-dimensional Context-aware Adaptation. Proc. of EICS'2011 (Pisa, 13-16 June 2011), ACM Press, New York, (2011)

2. Dessart, Ch.-E., Motti, V., Vanderdonckt, J., Showing User Interface Adaptivity by Animated Transitions, In Proc. EICS'2011 (Pisa), ACM Press, New York, (2011), 3. Balme, L., Demeure, A., Calvary, G., Coutaz, J.: Sedan-Bouillon: A Plastic Web Site. In: PSMD 2005, INTERACT 2005 Workshop on Plastic Services for Mobile Devices, (2005)

4. Grolaux, D.; Vanderdonckt, J.; \& Roy, P v. "Attach Me, Detach Me, Assemble Me Like You Work". Interact, 2005, LNCS 3585, pp. 198-212.

5. Brusilovsky, P. \& Schwarz, E. "ELM-ART: An Intelligent Tutoring System on the WWW" 